\title{
Praileaitz I (Deba, Gipuzkoa): el estudio paleopalinológico
}

\author{
Praileaitz I (Deba, Gipuzkoa): ikerketa palinologikoa \\ Praileaitz I (Deba, Gizpuzkoa): palaeopalynological analysis
}

PALABRAS CLAVE: Paleopalinología, Praileaitz I, Pleistoceno Superior, Paleolítico.

GAKO-HITZAK: Palinologia, Praileaitz, Goi Pleistozenoa, Paleolitikoa.

KEYWORDS: Palynology, Praileaitz I, Upper Pleistocene, Palaeolithic.

\section{María José IRIARTE-CHIAPUSSO(1, 2)}

\section{RESUMEN}

La conservación esporopolínica en la cueva de Praileaitz I es deficiente. Por este motivo, pese a haber analizado 65 muestras sólo hemos recuperado información de una muestra del nivel de ocupación solutrense. Esta puntual visión refleja un paisaje abierto con pocos representantes arbóreos (Pinus, Juniperus y Betula).

\section{LABURPENA}

Praileaitz I kobazuloan eman den palinomorfoen kontserbazioa nahiko kaskarra izan da. Nahiz eta 65 lagin analizatu egin direla, bakarrik batetan aurkitu izan dira emaitza esanguratsuak, Solutre aldiko maila batetik datorrena. Lagin honetan ikusten den paisaia oso zabala da, oso zuhaitz gutxirekin (Pinus, Juniperus eta Betula).

\section{ABSTRACT}

The preservation of palinomorphs in the cave of Praileaitz I is not good. Although a total amount of 65 samples has been analyzed, just one of them, from the Solutrean level, has givel useful information. This single sample gives us a view of an open landscape, with few arborean elements (Pinus, Juniperus and Betula).

\section{1.- INTRODUCCIÓN}

La cueva de Praileaitz I se sitúa en la cuenca baja del río Deba, en la ladera N-NE del monte Praileaitz. En este área geográfica del valle del Deba se concentran diversos yacimientos paleolíticos, como Ermittia, Iruroin, Langatxo

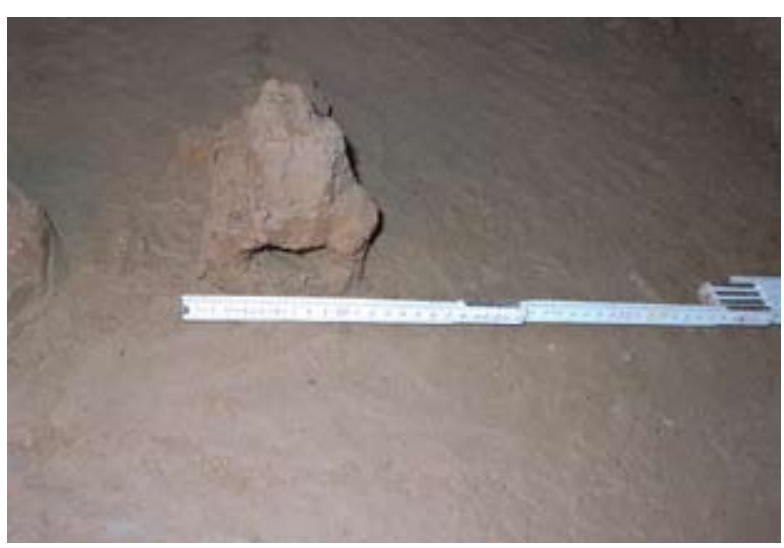

Fig. 1. Columna 3: cuadro 18E' (muestra 1). / Column 3: square $18 \mathrm{E}$ ' (sample 1). o Praileaitz II. La cavidad fue descubierta en 1983 por miembros del Grupo Munibe de Azkoitia, siendo a partir del año 2000 cuando comienza la excavación arqueológica bajo la dirección de Xabier Peñalver.

En esta cueva, cuya entrada actual ha estado condicionada por el desprendimiento de un gran bloque, se han diferenciado dos zonas de habitación, una exterior con escasos restos arqueológicos y otra interior donde se han detectado diversas ocupaciones del Paleolítico Superior, correspondiendo la más antigua al Gravetiense. La secuencia arqueológica se localiza en el vestíbulo y en la primera sala, a la que se accede tras un reducido pasillo, que también conduce a otros espacios.

\section{2.- EL ESTUDIO PALEOPALINOLÓGICO}

\subsection{Muestreo}

El muestreo de este depósito se ha realizado en coordinación con el progreso de la actuación arqueológica. Por ejemplo, en el caso de la columna 3 (cuadro 18 E') se recogieron en primer lugar las dos muestras más

\footnotetext{
(1) Área de Prehistoria - Facultad de Letras. Universidad del País Vasco UPV/EHU, c/Tomás y s/n, 01006, Vitoria-Gasteiz.

(2) IKERBASQUE, Basque Foundation for Science, 48011, Bilbao. mariajose.iriarte@ehu.es
} 


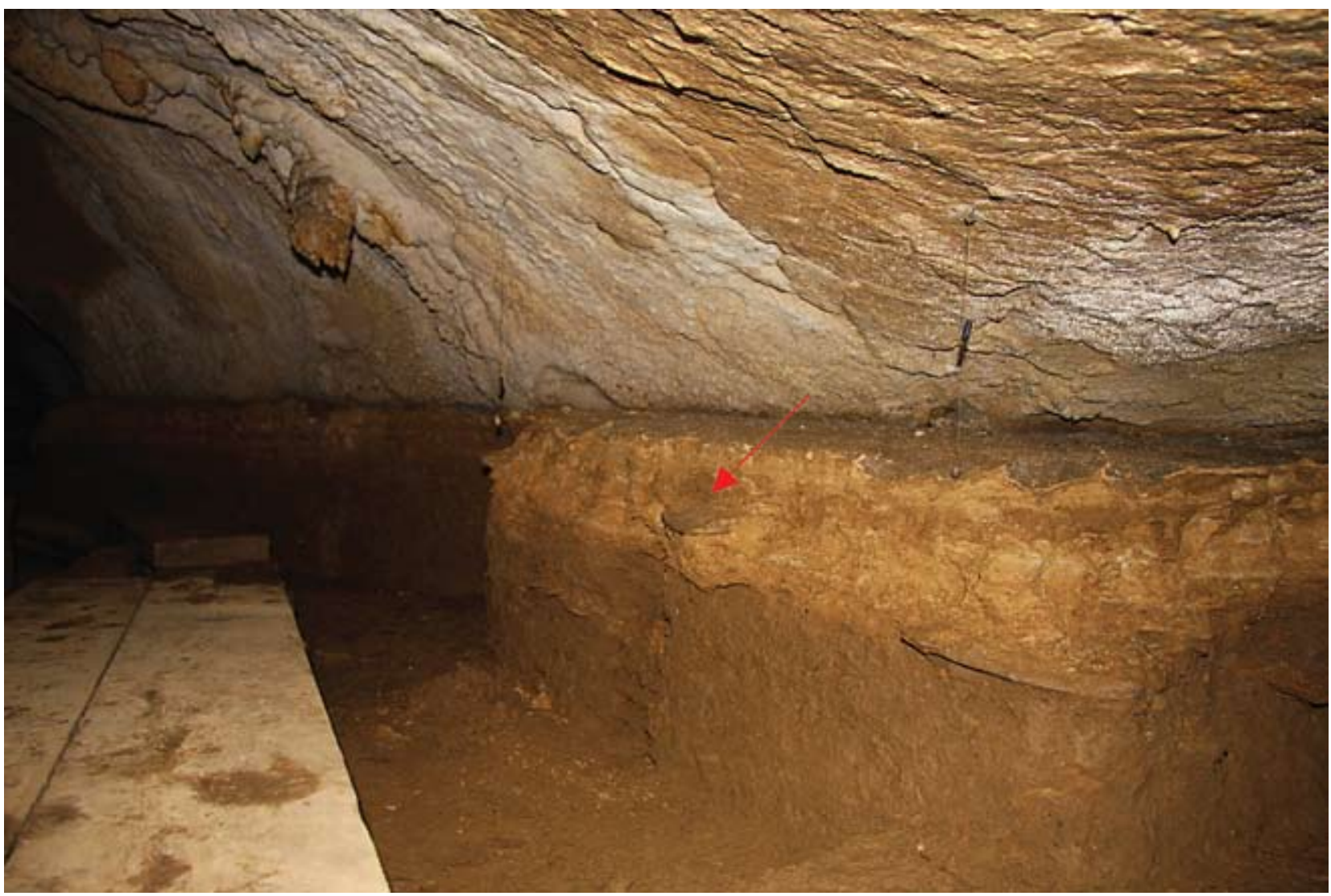

Fig. 2. Columna 6: cuadro 10J'. / Column 6: square 10J'.

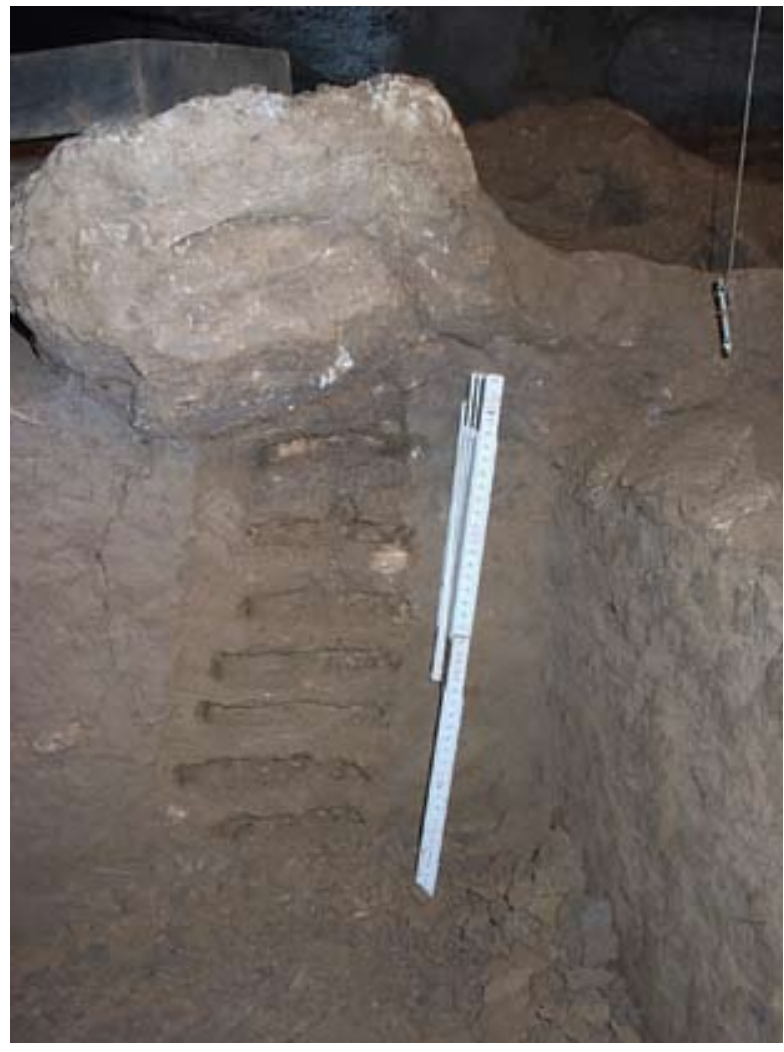

recientes, para no entorpecer la dinámica de la excavación. Una vez se fue profundizando más, en el mismo sitio en el que se situaban las muestras ya obtenidas, se reservó un pequeño testigo del que se obtuvo la muestra más antigua de esta columna (Fig. 1). Las muestras fueron tomadas en columna (sistema seleccionado en todos los muestreos de la cueva), tras la selección y limpieza del corte estratigráfico elegido (Figs. 2 y 3). En cada una de las muestras se recogió una cantidad aproximada de $100 \mathrm{gr}$ de sedimento, estando condicionado el intervalo de muestreo en algunas ocasiones por la presencia de clastos en el mismo. La toma de muestras se ha realizado en diferentes zonas de la cueva atendiendo en primer lugar a las características de la cavidad y a la representación de la totalidad de la secuencia arqueológica, y en segundo lugar, ante los resultados negativos obtenidos en las primeras columnas analizadas, con la finalidad de determinar si este problema de conservación esporopolínica se concentra en una zona específica de la cueva o si, por el contrario, obedece a un proceso tafonómico más generalizado.

En la figura 4 se observa la distribución de las diferentes columnas palinológicas en las que se han recogido un total de 65 muestras:

Fig. 3. Columna 5: cuadro 12G'. / Column 5: square 12G'. 


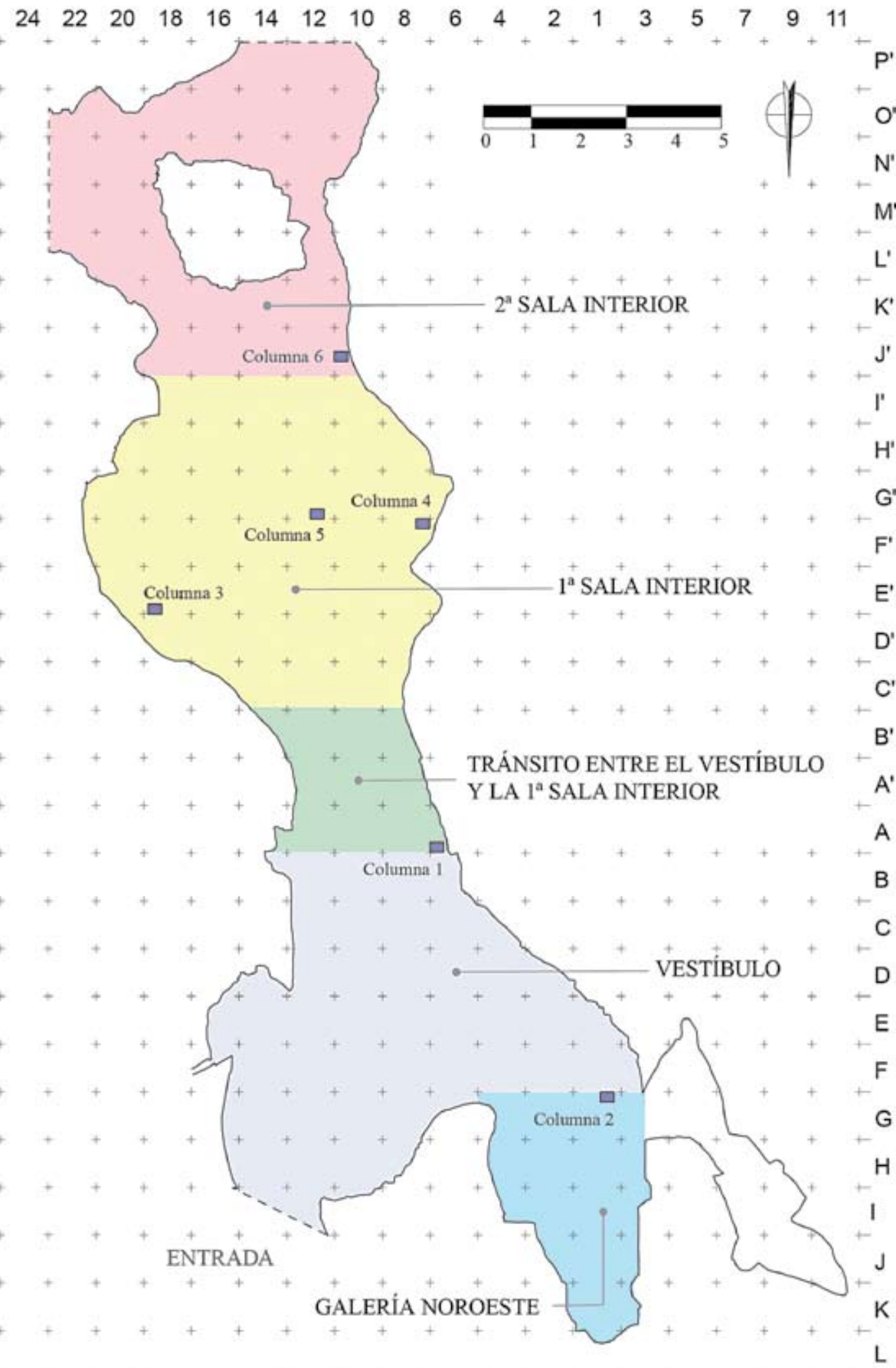

$\begin{array}{llllllllllllllllll}24 & 22 & 20 & 18 & 16 & 14 & 12 & 10 & 8 & 6 & 4 & 2 & 1 & 3 & 5 & 7 & 9 & 11\end{array}$

Fig. 4. Ubicación de las columnas palinológicas. / Location of palinological columns. 


\section{- columna 1 (cuadro 6A)}

muestras 1 a 6

muestras 7 a 10

muestras 11 a 21

- columna 2 (cuadro $1 \mathrm{G}$ )

muestra 1

muestras 2 a 9

muestras 10 y 11

muestras 12 a 21

\section{- columna 3 (cuadro 18 E')}

muestras 1 a 3

- columna 4 (cuadro 8 F')

muestra 1

muestra 2

\section{- columna 5 (cuadro 12 G')}

muestras 1 a 5

muestra 6

muestra 7

\section{- columna 6 (cuadro 10 J')}

muestras 1 y 2

muestras 3 a 8

\subsection{Trabajo de laboratorio}

El trabajo de laboratorio se inicia con el tratamiento físico-químico del sedimento para recuperar el contenido esporopolínico del mismo. En el tratamiento químico se ha empleado ácido clorhídrico para eliminar los carbonatos, e hidróxido sódico (reacción en caliente) para que actúe sobre los ácidos húmicos. Con la finalidad de separar el contenido polínico se utiliza el método de concentración del polen en licor denso (licor de Thoulet). Una vez filtrado, y eliminado el filtro, se procede al montaje del contenido esporopolínico recuperado en los respectivos portas para su identificación al microscopio (BURJACHS et al., 2003).

La determinación polínica y el recuento de palinomorfos se han efectuado mediante microscopía óptica (objetivos de 40x, 60x y 100x con aceite de inmersión). Los registros polínicos con un número mínimo inferior a 150-200 pólenes y un número inferior a 15 taxones se consideran polínicamente estériles, siendo aquellos que cumplen estas condiciones los estadísticamente válidos. En esta ocasión, los resultados se exponen en tablas sin incluir valores porcentuales.

\section{3.- RESULTADOS}

Los resultados obtenidos en las columnas polínicas han sido muy escasos, dado el pequeño número de pa- nivel $\mathrm{V}$ - Solutrense

nivel IV - Magdaleniense Inferior

nivel II - Epipaleolítico

nivel VII

nivel VI

nivel IV - Magdaleniense Inferior

nivel II - Epipaleolítico

nivel IV - Magdaleniense Inferior

nivel $\mathrm{V}$ - Solutrense

nivel IV - Magdaleniense Inferior

niveles VII y VI

nivel IV - Magdaleniense Inferior

nivel II - Epipaleolítico

nivel IX - Solutrense

nivel II - Epipaleolítico

linomorfos recuperados. Las dos columnas con mayor número de muestras (cuadros $6 \mathrm{~A}$ y $1 \mathrm{G}$ ) presentan un grado de conservación de los pólenes y esporas totalmente deficiente, siendo la mayoría de las muestras totalmente estériles. En aquellos casos en los que se han conservado algunos restos, éstos son tan escasos que no permiten obtener ningún tipo de conclusiones definitivas. Por ejemplo, en la muestra 8 (lecho 11 del nivel IV: Magdaleniense Inferior) solo se han recuperado dos pólenes de avellano y dos esporas de helecho tipo monolete (Fig. 5).

Al realizar el estudio de la columna del cuadro 18E', dada su localización en una zona diferente de la cavidad, pensamos en la posibilidad de obtener mejores resultados. Sin embargo, nuevamente nos encontramos con la misma situación. En estas muestras únicamente se ha recuperado un polen de gramínea (muestra 2), caracterizándose las otras muestras por la presencia de microcarbones dispersos y una esterilidad esporopolínica absoluta. Esta circunstancia se ha repetido en las muestras de la columna 4 (cuadro 8F').

Algunos resultados se han obtenido en algunas zonas de las columnas 5 y 6 , aunque en ningún caso se ha podido alcanzar los 125 pólenes por muestra. En el cuadro 12G' de la primera sala interior (columna 5) la información parcial disponible corresponde al nivel situado debajo del 


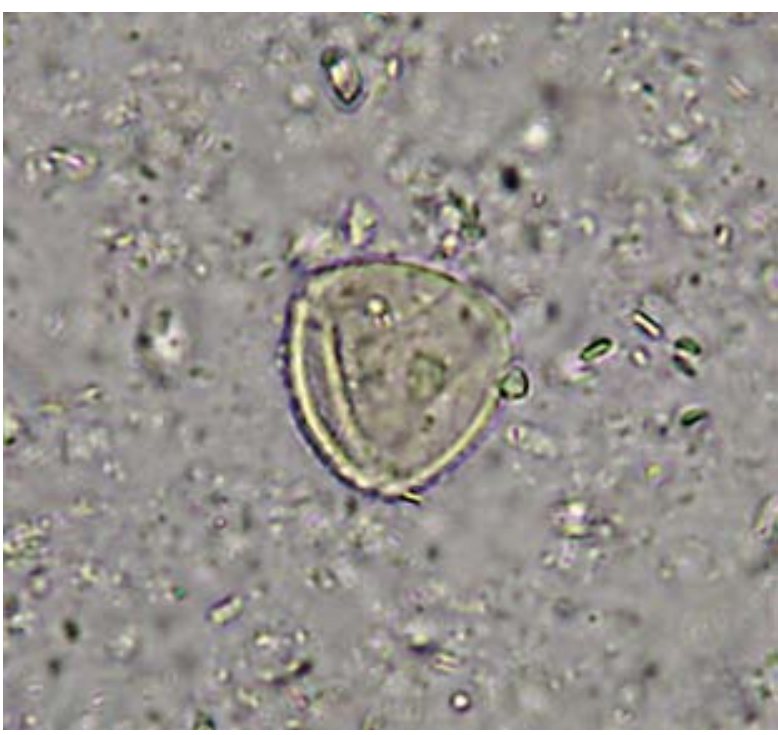

Fig. 5. Polen de avellano (columna 1 - muestra 8). / Hazel pollen (column 1 - sample 8). nivel IV (Magdaleniense Inferior). En este nivel la muestra 4 es la que más contenido esporopolínico tiene:

Polen arbóreo:

Pinus sp. (3 pólenes)

Polen no arbóreo:

Poaceae (9 pólenes)

Ericaceae (2 pólenes)

Compositae liguliflora (31 pólenes)

Compositae tubuliflora (3 pólenes)

Ranunculaceae (5 pólenes)

Esporas de helechos:

Filicales monolete (18 pólenes)

Filicales trilete (8 pólenes)

Polypodiaceae (7 pólenes)

Indeterminables (12 pólenes)

\begin{tabular}{|c|c|c|c|c|c|c|c|}
\hline & & \multicolumn{4}{|c|}{ C $u$ a $\quad d \quad r \quad o$} & 0 & , \\
\hline & & $\mathrm{m}_{\mathrm{S}}{ }^{1}$ & $\begin{array}{c}\mathrm{m} .3 \\
\mathrm{Ep}\end{array}$ & $\begin{array}{c}\mathrm{m} .4 \\
\mathrm{Ep}\end{array}$ & $\begin{array}{c}\mathrm{m} .5 \\
\mathrm{Ep}\end{array}$ & $\begin{array}{c}\mathrm{m} .6 \\
\mathrm{Ep}\end{array}$ & $\begin{array}{c}\mathrm{m} .7 \\
\mathrm{Ep}\end{array}$ \\
\hline \multirow{3}{*}{ 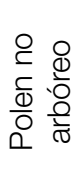 } & Poaceae & - & - & 1 & - & - & - \\
\hline & Compositae liguliflora & 2 & - & - & - & - & - \\
\hline & Polygonum & - & - & 1 & - & - & - \\
\hline \multirow{3}{*}{ 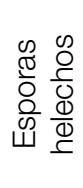 } & Filicales monolete & - & - & 1 & - & 1 & - \\
\hline & Filicales trilete & 1 & - & - & - & - & \\
\hline & Polypodium & 1 & - & - & - & - & - \\
\hline Ind. & & 3 & 1 & - & - & - & - \\
\hline
\end{tabular}

Tabl. 1. muestras totalmente estériles (o casi) de la columna 6. (S: Solutrense -nivel V- /Ep: Epipaleolítico -nivel II-). / Sterile (or almost) samples of column 6 (S: Solutrean -level V- / Ep: Epipalaeolithic -level II-).

Este registro polínico no resulta estadísticamente válido aunque tenga un mayor número de pólenes y cierta diversidad taxonómica. Ésta, es insuficiente para poder ser considerada representativa de las comunidades vegetales que existieron en el entorno del depósito. En este contexto, el predominio de las compuestas, familia caracterizada por tener una capa externa del polen (exina) más resistente al deterioro postdeposicional que otros taxones polínicos, podría estar relacionado con una conservación diferencial de los palinomorfos.

La última columna (cuadro 10J') mantiene la misma tónica que el resto: presencia de microcarbones disper- sos; muestras totalmente estériles o casi (Tabl. 1) y dos muestras con cierto contenido esporopolínico (Tabl. 2).

El espectro polínico de la muestra 2 de esta columna (nivel IX: Solutrense) destaca por la cantidad de pólenes y esporas que conserva (102 y 28 respectivamente), aunque, al igual que sucedía en la muestra 4 de la columna 5, numéricamente este registro no resulta estadísticamente válido y la diversidad taxonómica resulta insuficiente (este aspecto se agrava en la muestra 8 de esta misma columna).

A pesar de estas limitaciones, este espectro polínico nos aporta información sobre el paisaje vegetal de esta 


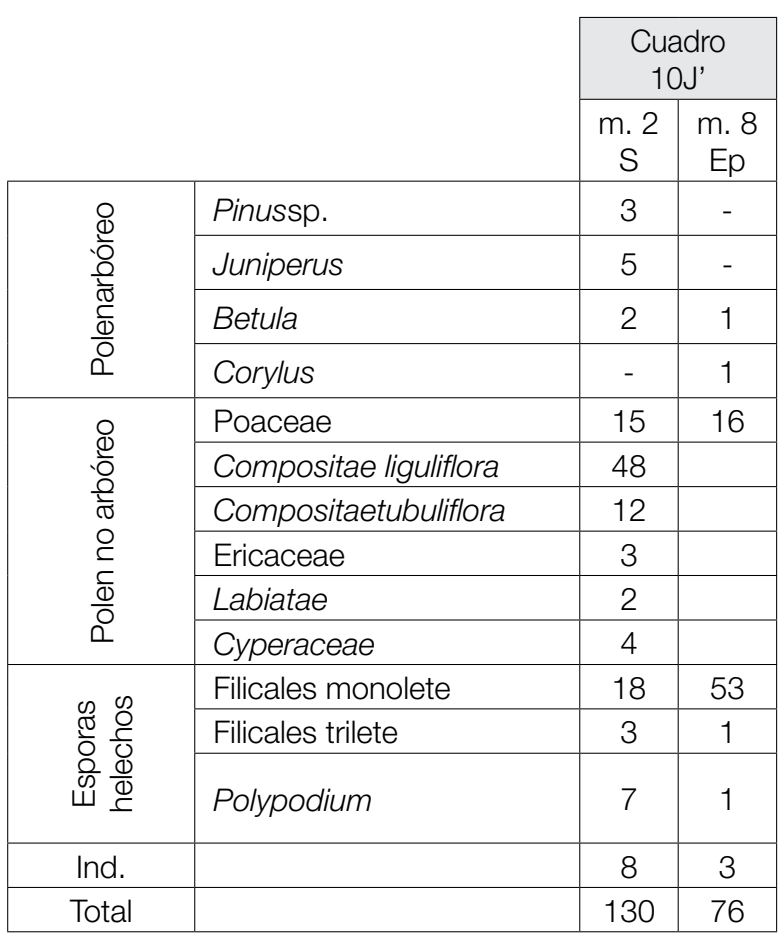

Tabl. 2. muestras de mayor contenido esporopolínico de la columna 6. (S: Solutrense -nivel V- / Ep: Epipaleolítico -nivel II-). / Greater pollen content samples in column 6 (S: Solutrean -level V- / Ep: Epipalaeolithic -level II-).

zona del valle del Deba, en torno al 17.500 BP. Los tres taxones arbóreos presentes (pino, enebro y abedul) indican unas condiciones climáticas poco favorables. Una característica común, observada en los periodos de mayor deterioro climático de otras secuencias paleoambientales solutrenses de la Región Cantábrica, es la existencia de paisajes abiertos con una reducida cubierta arbórea en la que el pino suele ir acompañado del enebro y/o abedul (por ejemplo: nivel 8 de la cueva de La Riera -LEROI-GOURHAN, 1986-; nivel paleontológico Arg-o de la cueva de Santimamiñe -IRIARTE-CHIAPUSSO, 2011-; nivel paleontológico F de la cavidad de Kiputz IX -GARCÍA-IBAIBARRIAGA et al., 2013)-. Por el contrario, dentro de la horquilla cronológica en la que se desarrolló el Solutrense, también existieron periodos de mejoría climática en la que las especies caducifolias (avellano, roble, aliso, olmo, etc.) adquieren un mayor protagonismo dentro del estrato arbóreo (nivel 3 de la cueva de Cobrante -RUIZ-ZAPATA y GIL GARCÍA, 2009-, nivel 1 de la cueva de Chufín -BOYER-KLEIN, 1980 y 1984-y zona superior del nivel IV de la cueva de Amalda -DUPRÉ 1990-). No obstante, incluso en estos momentos de recuperación, la masa forestal no ocupó grandes extensiones, situándose en torno al 10\% el valor medio del polen arbóreo (cueva Chufín y Cobrante tienen sendas excepciones al superarse el 20\%). Evidentemente, cuando las condiciones empeoran, la cubierta arbórea retrocede (valores inferiores al 5\%). En el caso del registro de
Praileaitz I, no es posible establecer el valor porcentual del estrato arbóreo.

Entre los escasos taxones herbáceos de la muestra 2, claramente destacan las compuestas (ligulifloras y tubulifloras) seguidas de las gramíneas (Poaceae). Anteriormente, ya se ha indicado que, dados los problemas de conservación esporolínica existentes en Praileaitz I, la razón de este predominio podría estar relacionado con una conservación diferencial de los pólenes. Sin embargo, en el contexto de esta muestra, no se puede descartar que las compuestas tuvieran un papel relevante dentro del estrato herbáceo porque se ha observado en otros depósitos (por ejemplo Amalda, El Mirón -STRAUS et al., 2011; STRAUS et al., 2013- o Santimamiñe) que, al producirse un deterioro en las condiciones climáticas, existe un aumento del polen de este taxon que suele coincidir con un descenso del grado humedad y de la representación de gramíneas y brezos (RAMIL-REGO et al., 2005, GARCÍA-IBAIBARRIAGA et al., 2013). En el caso de Praileaitz I, no podemos saber cómo evolucionó el grado de humedad ante la falta de datos sobre la dinámica de las esporas de helechos y de otros taxones higrófilos.

\section{4.- CONSIDERACIONES FINALES}

La cavidad de Praileaitz I tiene un problema generalizado de conservación esporopolínica. Independientemente de la zona del yacimiento donde hayamos muestreado, el resultado es el mismo: un deficiente grado de conservación de los pólenes y esporas que alcanza su máxima expresión en la gran cantidad de muestras en las que no se ha recuperado ningún resto.

Así, no se ha dispuesto de ningún registro estadísticamente válido de un total de 65 muestras analizadas. La única compensación obtenida es la pincelada que, sobre el entorno medioambiental de la ocupación solutrense en Praileaitz I, ha ofrecido la muestra 2 del cuadro 10J' de la segunda sala interior. Los reducidos datos recuperados, junto con la información procedente de otros depósitos, nos permite imaginarnos que los moradores de este sitio, desde la entrada de la cavidad, verían un paisaje abierto con unos pocos pinos, enebros y abedules, que contrasta con el frondoso bosque mixto caducifolio que cubre la ladera del monte Praileaitz en la actualidad.

\section{5.- BIBLIOGRAFÍA}

BOYER-KLEIN, A

1980 Nouveaux résultats cantabriques au Tardiglaciaire. Bulletin de la Societé Préhistorique Française 77/4, 103-107.

1984 Analyses polliniques cantabriques au Tardiglaciaire. Revue de Paleobiologie vol. spec., 33-39.

BURJACHS, F., LÓPEZ-SÁEZ, J.A., IRIARTE, M.J.

2003 Metodología arqueopalinológica, en BUXÓ, R. \& PIQUÉ R. (dir.) La recogida de muestras en arqueobotánica: ob- 
jetivos y propuestas metodológicas. 11-18. Museu d'Arqueologia de Catalunya. Girona.

DUPRÉ, M.

1990 Análisis polínico de la cueva de Amalda, en ALTUNA, J., BALDEON, A. \& MARIEZKURRENA, K. (eds.) La Cueva de Amalda (Zestoa, País Vasco). 49-51. Eusko Ikaskuntza. Donostia. (Colección Barandiarán; 4).

GARCÍA-IBAIBARRIAGA, N., IRIARTE-CHIAPUSSO, M.J., MUREALAGA, $X$.

2013 El registro micropaleontológico del Solutrense en la Región Cantábrica: estado de la cuestión. Congreso Internacional El Solutrense: Centenario de las excavaciones en La Cueva de Ambrosio (Velez-Almería).

IRIARTE-CHIAPUSSO, M.J.

2011 Polen y vegetación en la secuencia estratigráfica de Santimamiñe (Kortezubi, Bizkaia), en LÓPEZ QUINTANA, J.C. (dir). La cueva de Santimamiñe: revisión y actualización (2004-2006). 321-342. Diputación Foral de Bizkaia. Bilbao.

JORDÁ-PARDO, F.J., ALVAREZ-ALONSO, D., IRIARTE-CHIAPUSSO, M.J.

2014 Una aproximación geoarqueológica al hábitat humano pleistoceno del occidente cantábrico (Asturias, norte de España), en: ALVAREZ-ALONSO, D. (Ed.). Los grupos cazadores-recolectores paleolíticos del occidente cantábrico. Estudios en Homenaje a Francisco Jordá Cerdá en el centenario de su nacimiento. 1914-2014. 67-102. UNED Centro Asociado de Asturias. Gijón. (ENTEMU; XVIII).
LEROI-GOURHAN, ARL.

1986 The palynology of La Riera Cave, en STRAUS, L.G. \& CLARK, G.A. (eds.). La Riera Cave. 59-64. Tempe. (Anthropological Research Papers; 36).

RAMIL-REGO, P., IRIARTE, M.J., MUÑOZ-SOBRINO, C., GÓMEZ-ORELLANA, L.

2005 Cambio climático y dinámica temporal del paisaje y de los hábitats en las ecorregiones del NW de la Península Ibérica durante el Pleistoceno superior. Munibe Homenaje a J. Altuna 57(1), 537-551.

RUIZ-ZAPATA, B., GIL-GARCÍA, M.J.

2009 El paisaje vegetal de los alrededores de la cueva de Cobrante: análisis palinológico, en RASÍNES DEL RIO, P. (dir.). Arqueología en la Cueva de Cobrante (Cantabria, España). 55-62. (Sautuola; 15).

STRAUS, L.G., GÓNZALEZ, M.R., GUTIERREZ, I., IRIARTECHIAPUSSO, M.J.

2011 Further Solutrean evidence in El Mirón cave (Ramales de la Victoria, Cantabria). Munibe Antropología-Arkeologia $62,117-133$.

STRAUS, L.G., GONZALEZ MORALES, M.R., MARIN ARROYO, A.B., IRIARTE-CHIAPUSSO, M.J.

2013 The human occupations of El Miron cave (Ramales, Cantabria, Spain) during the last glacial maximum/solutrean period. Espacio Tiempo y Forma. Serie I, Prehistoria y Arqueología. Nueva época. "De punta a punta. El Solutrense en los albores del siglo XXl", 5. Congreso Internacional "El Solutrense - Centenario de las excavaciones en La Cueva del Ambrosio". Vélez-Blanco. Junio de 2012. 
